\title{
Fundamental constants in effective theory
}

\author{
G.E. Volovik \\ Low Temperature Laboratory, Helsinki University of Technology \\ P.O.Box 2200, FIN-02015 HUT, Finland
}

L.D. Landau Institute for Theoretical Physics

Kosygin Str. 2, 117940 Moscow, Russia

November 16, 2018

\begin{abstract}
There is a discussion between L. B. Okun, G. Veneziano and M. J. Duff, concerning the number of fundamental dimensionful constants in physics [1]. They advocated correspondingly 3, 2 and 0 fundamental constants. Here we consider this problem on example of the effective relativistic quantum field theory, which emerges in the low energy corner of quantum liquids and which reproduces many features of our physics including Lorentz invariance, chiral fermions, gauge fields and dynamical gravity.
\end{abstract}

\section{Introduction.}

Experimental bounds on possible variation of fundamental constants in nature are discussed in comprehensive review [2]. Since we live well below the Planck energy scale, we canot judge which of them are really fundamental, i.e. cannot be derived from the underlying trans-Planckian physics. Here we discuss this problem using the effective relativistic quantum field theory (RQFT) arising as emergent phenomenon in quantum liquids [3], or in other condensed matter systems [4. Since the trans-Planckian physics of a 
quantum liquid - the microscopic atomic physics - is well known at least in principle, this allows us to look at the problem of fundamental constants from the outside, i.e. from the point of view of an external observer who does not belong to the low-energy world of the effective RQFT. This observer knows both the effective and microscopic physics. He or she knows the origin of the 'constants' which enter the effective theory, and thus can judge whether they are fundamental within the effective theory, and whether they remain fundamental at the more fundamental (i.e. more microscopic) level.

The status of fundamental constants in quantum liquids depends on the energy scale used: the low-energy scale where the effective theory is applicable, or the atomic scale of the "Theory of Everything". Within the effective theory the status of constants also depends on the observer who measures them: the 'inner' observer belonging to the world of the low-energy quasiparticles, or the external observer who belongs to the world of microphysics. In Table (目) we shall use the following criteria of fundamentality. The first line shows whether $(+)$ or not $(-)$ the constant is fundamental from the point of view of an inner observer. The constant is fundamental if it cannot be derived within the effective theory used by the inner onbserver. The second line refers to the external observer if he uses the effective theory only. For him the fundamental constant is the phenomenological parameter. In complete equilibrium of a quantum liquid, and in the absence of external perturbations, this phenomenological parameter is completely fixed and thus can be considered as a fundamental. Finally the third line corresponds to Weinberg criterion [5]: the constant is not fundamental if it can be derived from the parameters of microscopic physics.

In the Table we consider the status of following constants: the Planck constant $\hbar$; the speed of light $c$, i.e. the maximum attainable velocity in the effective theory; and the Newton's gravitational constant G. From 19 parameters of the Standard Model we discuss only one - the fine structure constant $\alpha$, which has a direct analog in superfluid ${ }^{3}$ He- $\mathrm{A}$ [3].

$\begin{array}{ccccc}\text { 'constant' } & \hbar & G & c & \alpha \\ \text { effective theory }-- \text { inner observer } & + & + & + & - \\ \text { effective theory }-- \text { external observer } & + & + & - & - \\ \text { microphysics } & + & - & - & -\end{array}$

Let us now discuss the status of each of 4 constants. 


\section{Planck constant $\hbar$}

Inspection of the RQFT emerging in quantum liquids demonstrates that within this scheme we have only one fundamental constant - the Planck constant $\hbar$. It is fundamental both within the effective RQFT at low energy and in the microscopic physics of liquids (all signs in $\hbar$-column of Eq. (四) are + ). Quantum mechanics is built in quantum liquids, which are essentially quantum objects. It is the quantum zero point motion of atoms which gives rise to the ground state of the ${ }^{3} \mathrm{He}-\mathrm{A}$ liquid, where the low-energy fermionic and bosonic collective modes behave as relativistic chiral fermions, and gauge and gravitational fields. The limit $\hbar=0$ simply does not exist, since there is no vacuum at $\hbar=0$, and thus no effective theory.

In quantum liquids belonging to the same universality class of Fermipoints as ${ }^{3} \mathrm{He}-\mathrm{A}$, all physical laws of our world are more or less reproduced in the low-energy corner, except for quantum mechanics. In principle, it is not excluded that in the more comprehensive systems, quantum mechanics will also acquire the status of an emergent low-energy phenomenon [6], with the minus sign in the third line of the $\hbar$-column. Since this does not happen in our quantum liquids, we have no solid basis for discussion of the possibility of varying $\hbar$.

\section{Speed of light}

The metric of the effective 'Minkowski' space-time, in which the "relativistic" quasiparticles (fermions and bosons) propagate along the geodesic curves in anisotropic quantum liquids, has the following local structure in case of anisotropic quantum liquid: $g_{\mu \nu}=\operatorname{diag}\left(-1, c_{x}^{-2}, c_{y}^{-2}, c_{z}^{-2}\right)$. The analog of speed of light - the maximum attainable speed for low-energy fermions or/and bosons - depends on the direction of their propagation. Since for an external observer there is no unique speed of light $c$, we have the minus sign in the second and third lines of the $c$-column in Eq. (11).

The question, which arises in case of the anisotropic speed of light: What 'speed of light' enters the Einstein relation $M=m c^{2}$ between the rest energy and the mass of the object? More generally: Which $c$ enters physical equations?

The answer to this question is rather simple: the speed of light $c$ never 
enters explicitly any physically reasonable equation if it is written in covariant and gauge invariant form. The speed of light is hidden within the metric tensor, which is the relevant dynamical variable. For example, the above metric $g_{\mu \nu}=\operatorname{diag}\left(-1, c_{x}^{-2}, c_{y}^{-2}, c_{z}^{-2}\right)$ enters the energy spectrum of massive particle in the following way: $E^{2}=M^{2}+g^{i k} p_{i} p_{k}$, or $g^{\mu \nu} p_{\mu} p_{\nu}+M^{2}=0$, where $M$ is the rest energy. In these equations there is no $c$.

Usually the mass is determined as the response of momentum to velocity: $p_{i}=M_{i k} v^{k}$. Since the velocity of the particle is $v^{i}=d E / d p_{i}$, one obtains for the mass tensor: $M_{i k}=E g_{i k}$. If the linear reponse of the momentum to velocity is considered one has

$$
M_{i k}(\text { linear }) \equiv M_{i k}(\mathbf{v}=0)=M g_{i k} .
$$

This equation contains the rest energy $M$, but does not contain $c$ and $m$ explicitly. The same is valid for any equation which is written in covariant form: they never contain $m$ and $c$ separately.

In the effective theory, the problem of the fundamenatlity of $c$ is rather peculiar. For an inner observer living in the liquid, who uses the rods and clocks made of low-energy quasiparticles, the "speed of light" does not depend on the direction of propagation. Moreover it does not depend on the velocity of the inner observer with respect to the liquid. This happens because of the physical Lorentz contraction experienced by his rods made of quasiparticles; his clock made of quasiparticles experience the retardation of time. All this conspire to make the inner observer to believe that the speed of light is fundamental. Such low-energy observer can savely divide the rest energy $M$ by his $c^{2}$. He will obtain what he thinks is the mass of the object, and this belief will be shared by all the low-energy inner observers. In this sense the speed of light is fundamental and the first line of Table (11) contains the plus sign.

However, for the external observer who lives outside the quantum liquid and uses the rigid rods and laboratory clocks in his experiments, the speed of massless quasiparticles is anisotropic even in the low-energy limit, i.e. in the range of applicability of the effective theory. He or she finds that, say, in ${ }^{3} \mathrm{He}-\mathrm{A}$ the "speed of light" varies from $3 \mathrm{~cm} / \mathrm{s}$ to $60 \mathrm{~m} / \mathrm{s}$ depending on the direction. This means that for external observer the speed of light is not fundamental both in the microscopic and effective theories (the minus sign both in the second and third lines of (11)). 


\section{Newton's constant}

In some (very special) cases [3] the action for the effective gravitational field in quantum liquids is similar to the Einstein action

$$
S_{\text {Einstein }}=-\int d^{4} x \frac{\sqrt{-g}}{16 \pi G} \mathcal{R}
$$

As in the case of Sakharov's induced gravity [9], the analog of the gravitational constant $G$ in quantum liquids is determined by the Planck energy cut-off $E_{\text {Planck }}$ (played by the amplitude of the superfluid gap $\Delta_{0}$ ) and by the number of the chiral fermionic species: $G^{-1} \sim N_{F} E_{\text {Planck }}^{2} / \hbar$. Since $G$ is determined by the microscopic physics, it is not fundamental on the microscopic level - minus sign in third line.

In quantum liquids the effective $G$ depends on temperature [3], $G^{-1}(T)=$ $G^{-1}(0)+\gamma N_{F} T^{2} / \hbar$, where $\gamma$ is the dimensionless factor of order unity. Since the temperature correction does not contain the microscopic parameters and thus in principle can be obtained within the effective theory, $G$ is not fundamental even on the effective level. But its vacuum value $G(T=0)$ is fundamental for an inner observer.

Moreover, the phenomenological parameter $G(T=0)$ of the effective theory as viewed by the external observer has a definite value for the quantum liquid isolated from the environment. That is why it can be considered as the fundamental constant of the effective theory, whence the plus sign in the second line of $G$-column.

\section{Fine structure constant}

In logarithmic approximation, the action for effective electrodynamics emerging in ${ }^{3} \mathrm{He}-\mathrm{A}$ (and in other systems of the Fermi-point universality class) is [3]:

$$
S_{\mathrm{em}}=\frac{1}{\hbar} \int d t d^{3} x \frac{\sqrt{-g}}{16 \pi \alpha} F^{\mu \nu} F_{\mu \nu} .
$$

An analog of the fine structure constant $\alpha$ is not fundamental both on microscopic and effective levels, since it logarithmically depends both on ultraviolet and on infra-red cut- off parameters. If the largest infra-red cut-off is sup-

plied by temperature, one has $\alpha^{-1} \sim N_{F} \ln \left(E_{\text {Planck }} / T\right)$, where $N_{F}$ is again the 
number of chiral fermionic species, and the ultraviolet cut-off is determined by the same Planck scale $E_{\text {Planck }}=\Delta_{0}$ which enters the effective Newton's constant [3]. It is the same effective electrodynamics as was discussed by Zeldovich [7]. Dependence on $E_{\text {Planck }}$ shows that $\alpha$ is not fundamental on the microscopic level; the dependence on the low-energy scale demonstrates that it is not fundamental within the effective theory too. In contrast to Newton's constant, in the limit of $T=0$ and zero mass of all the Standard Model fermions the constant $\alpha$ vanishes. That is why the $\alpha$-column contains only the minus signs in Eq. (11).

Equation 1 does not contain speeds of light $c_{x}, c_{y}$ and $c_{z}$ explicilty: these functions are hidden in the metric field. The anisotropic speed of light is the crucial argument that the decomposition of the dimensionless running coupling $\alpha$ into dimensionful $e^{2}, \hbar$ and $c$ is meaningless: $e^{2}$ and $c$ do not enter explicitly any covariant and gauge invariant equations. For example, the energy levels of the electron in the hydrogen atom is determined by $\alpha$ and by the rest energy $M_{e}$ of the electron [8]:

$$
E_{n, j}=M_{e}\left[1-\frac{\alpha^{2}}{2 n^{2}}-\frac{\alpha^{4}}{2 n^{4}}\left(\frac{n}{j+1 / 2}-\frac{3}{4}\right)+\ldots\right]
$$

Let us recall that in contrast to the quantity $e$, the physical electric (or other) charge of elementary particles in Standard Model and its analog in ${ }^{3} \mathrm{He}-\mathrm{A}$ are dimensionless (integer or fractional) quantities determined by geometry. They are determined either by gauge groups in fundamental qauge theories or by momentum-space topology in quantum vacua of the Fermi-point universality class.

The only fundamental constant which enters explicitly the electromagnetic action is $\hbar$.

\section{What constants are really fundamental?}

Thus, in our example of the relativistic quantum field theory arising in quantum liquids, the only fundamental constant is $\hbar$. Since it is fundamental it is possible to use it as a conversion factor of energy to frequency as is stated by Duff in [1] (of course, with the following reservations: (i) energy is additive while frequency is not; (ii) as distinct from frequency the magnitude of energy depends on the choice of zero energy level; (iii) the frequency can be 
bounded from above due to, say, discreteness of time, while the energy is not bounded: it can be the sum of energies of many low-energy particles; etc.).

However, it is not completely excluded that $\hbar$ can arise as a result of a more fundamental theory above the Planck scale which gives rise to the quantum mechanics in the low-energy scale [6].

In such case the only fundamental constants which remain in the effective RQFT are the dimensionless charges and dimensionless topological quantum numbers [10, 3] which enter the topological terms in effective action. Such terms are naturally dimensionless, since they are determined either by gauge groups or by the topological invariants in real and in momentum spaces. In $3+1$ systems, such invariants describe the chiral anomaly [11, 12], and other anomalies. In $2+1$ systems, these topological quantum numbers lead to quantization of physical parameters such as Hall and spin Hall conductivities, which being written in the covariant form are integer of rational numbers. 


\section{References}

[1] M. J. Duff, L. B. Okun, and G. Veneziano, Trialogue on the number of fundamental constants, JHEP 03023, 1-30 (2002), physics/0110060.

[2] J.-P. Uzan, The fundamental constants and their variation: observational status and theoretical motivations, Rev. Mod. Phys. to be published, hep-ph/0205340.

[3] G.E. Volovik, Superfluid analogies of cosmological phenomena, Physics Reports, 351, 195-348 (2001); Universe in a Helium Droplet, forthcoming book, Oxford University Press, http://ice.hut.fi/Volovik/book.pdf.

[4] R.B. Laughlin and D. Pines, The Theory of Everything, Proc. Natl. Acad. Sc. USA 97, 28-31 (2000).

[5] S. Weinberg, Overview of theoretical prospects for understanding the values of fundamental constants, in: The Constants of Physics, W.H. McCrea and M.J. Rees editors, Phil. Trans. R. Soc. London A 310, 249 (1983).

[6] S.L. Adler, Statistical dynamics of global unitary invariant matrix models as pre-quantum mechanics, hep-ph/0206120.

[7] Ya.B. Zel'dovich, Interpretation of electrodynamics as a consequence of quantum theory, Pis'ma ZhETF 6, 922 (1967); [JETP Letters, 6, 345 (1967)].

[8] S. Weinberg The Quantum Theory of Fields, Cambridge University Press (1995).

[9] A.D. Sakharov, Dokl. Akad. Nauk SSSR, 177, 70 (1967); [Sov. Phys. Doklady 12, 1040 (1968)].

[10] D.J. Thouless Topological Quantum Numbers in Nonrelativistic Physics, World Scientific (1998).

[11] S. Adler, Axial-vector vertex in spinor electrodynamics, Phys. Rev. 177, 2426 - 2438 (1969). 
[12] J.S. Bell and R. Jackiw, A PCAC puzzle: $\pi_{0} \rightarrow \gamma \gamma$ in the $\sigma$ model, Nuovo Cim. A 60, 47-61 (1969). 